\title{
AC 2009-1218: DEVELOPMENT OF A NANOSCALE VIRTUAL ENVIRONMENT HAPTIC INTERFACE FOR TEACHING NANOTECHNOLOGY TO INDIVIDUALS WHO ARE VISUALLY IMPAIRED
}

Dianne Pawluk, Virginia Commonwealth University

Currently an Assistant Professor in the Department of Biomedical Engineering, VCU.

\section{Curtis Taylor, University of Florida}

Currently an Assistant Professor in the Department of Mechanical and Aerospace Engineering, University of Florida.

Marcia Hoffman, Virginia Commonwealth University

Completed her undergraduate degree in Biomedical Engineering at VCU.

Maria McClintock, Virginia Commonwealth University

Completing her undergraduate degree in engineering at VCU. 


\title{
Development of a Nanoscale Virtual Environment Haptic Interface for Teaching Nanotechnology to Individuals who are Visually Impaired
}

\begin{abstract}
Nanotechnology is a relatively new, exciting and growing area of research in which governments, educators and researchers, alike, are interested in attracting K-12 and undergraduate students to pursue future careers. However, how things interact at the small scale of a nano-environment can be difficult for these students to understand and conceptualize. This is particularly true for students who are visually impaired, as most current explanations and pedagogical methods heavily rely on $2-\mathrm{D}$ visual diagrams or molecular-scale images. These methods are also potentially limited in their engagement of sighted students, whom have a diversity of learning styles. Therefore, there is a need for new methods, for both visually impaired and sighted students, to aid in their engagement to understand nanoscale concepts and to pique their interest about the field of nanotechnology. In this paper, we describe the development of a haptic feedback system interfaced with a nanoscale virtual environment to facilitate exploration, perception and conceptualization of nanoscale science and engineering concepts. The system is especially accessible to individuals who are visually impaired since, through haptics, it provides a physical mechanism to aid in perception and conceptualization of nanoscale forces and objects. An overview of learning modules and instructional material for use with the haptic interface are discussed.
\end{abstract}

The current state-of-the-art in the use of haptics for nanotechnology has focused on high-end $(>\$ 10,000)$ devices for real-time nano-telemanipulation of nanoscale microscopy instrumentation such as an atomic force microscope (AFM) or visualization of complex (molecular scale) research simulations. Here we consider a more widely accessible avenue for $\mathrm{K}-12$ and undergraduate instruction: using a relatively inexpensive force feedback device, the Novint Falcon Force Feedback Game Controller (\$189) inside a virtual environment. This makes the instructional material cost effective, easier to use, and amenable to implementation in high school and college classrooms. It also allows a student to easily switch between experiencing the macroscale and nanoscale for comparison purposes, facilitating the learning process by allowing them to relate the nanoscale to something they are familiar with.

Two modules have been developed introducing nano-topologies and nano-forces to students in comparison to the macro-world. For the actual implementation on the Novint Falcon, both the macro- and nano-surface topologies are simulated using an analog of AFM--a relatively stiff virtual spring is used to model haptic contact of a probe with the surface. The student can then move the probe-shaped handle of the Falcon to follow the topology of the hard surfaces. For sighted students, the corresponding visual graphics are also presented. For describing the interaction forces, mathematical equations are used directly to describe a probe tip interacting with a flat surface, with gravity and inertia dominating at the macroscale, and van der Waals and electrostatic forces dominating at the nanoscale. The student is able to move the Falcon towards and away from the surface, at the different scales, to feel the contribution of the different forces. They are also able to "turn-off" components of the net force to enable understanding of the effects of individual types of forces. 


\section{Introduction}

Nanotechnology is a relatively new, exciting and growing area of research in which governments, educators and researchers, alike, are interested in attracting K-12 and undergraduate students to pursue future careers. However, things interact on the nano-level in a very different way than in the macro world, and this can be very difficult for students to understand and conceptualize. This is particularly true for students who are visually impaired as most current explanations and pedagogical methods heavily rely on 2-D visual diagrams or molecular-scale images. These methods are also potentially limited in their engagement of sighted students, whom have a diversity of learning styles. Therefore, there is a need for new methods, for both visually impaired and sighted students, to aid in their engagement to understand nanoscale concepts and to pique their interest about the field of nanotechnology.

To achieve this aim, we propose presenting information about nanotechnology haptically, in a virtual reality system that simulates both the macro and nano-worlds. Haptics is the field of perception that consists of the combination of the sense of kinesthesia (i.e., perception of forces and joint motion) and the sense of touch. Haptic technology can involve both force feedback devices (i.e., that produce controlled forces at the endpoint of the haptic device) and/or tactile

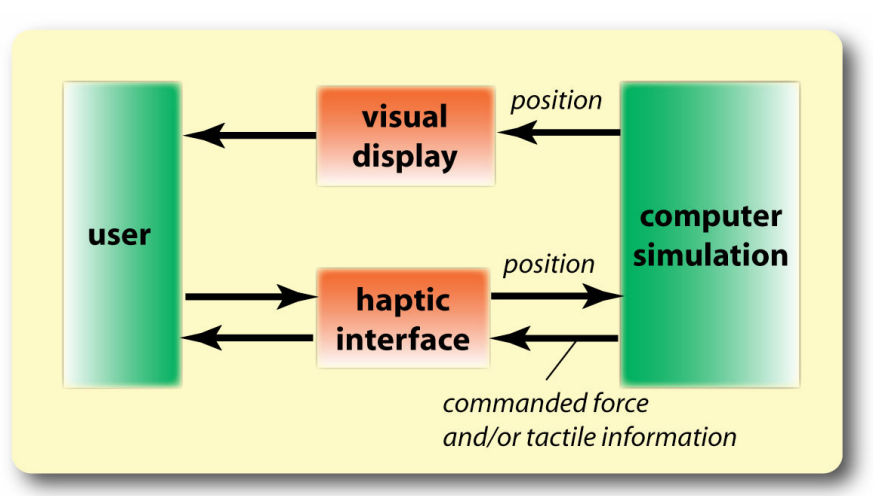

Figure 1. Block diagram of a virtual reality system. feedback devices (e.g., that produces vibrations, surface contact geometry or roughness). For a virtual reality system, the components consist of that shown in Figure 1; the system may or may not include a visual component. Obviously haptic feedback is very important for communicating physical ideas to individuals who are visually impaired. It is also the most intuitive method in instructing individuals about the forces involved in a physical system, as students can directly feel the forces involved.

Several different researchers have developed ${ }^{4,6-8}$ or used ${ }^{5,9}$, haptic interfaces for hands-on instruction of physics, engineering statics, dynamics and control systems in undergraduate curricula. Where evaluated, the perceived value by the students of using the haptic interfaces with virtual reality to teach concepts was relatively high and in all cases the students were excited about the use of haptic feedback.

For nanotechnology, many researchers (e.g., Sitti and his colleagues ${ }^{10}$ ) have examined the use of scaled haptic feedback in real-time from an atomic force microscope (AFM) to a haptic interface interacting with a user. Jones and her colleagues ${ }^{11}$ reported results that suggested students found the experience engaging, developed more positive attitudes toward science and showed significant gains in their understanding of the targeted topic (viruses). However, the costs would be prohibitive for the classroom (unless at a University with an existing AFM), the preparation would be difficult (unless the instructor was an expert in nanotechnology) and the experience would be effectively limited to one student at a time due to the cost of an AFM. The other 
disadvantage is that students would not be able to easily compare and contrast the macro to the nano world, which using a virtual environment would allow. A virtual environment would also allow students to selective turn on and off forces to see the effect of individual components.

More recently, two groups of researchers have examined the use of virtual reality for educating students about a specific nanoscale phenomenon: the approach-retraction forces of an AFM to a surface. Marchi and his colleagues ${ }^{14}$ compared the actual use of an AFM to a virtual reality simulator consisting of a combination of force, visual and auditory displays rendering the information. They found that the use of virtual reality was more effective at describing the phenomenon than the original AFM, possibly because it allowed the manipulation of parameters of the equations describing the forces. However, they did not directly examine the effectiveness of haptic feedback alone and their user group consisted of Master's degree students who spent three hours in focused study. Millet and his colleagues ${ }^{13}$ also explored the benefit of using haptic interfaces. Although they found that the use of haptics improved the students' understanding of the phenomenon, they did not examine whether this could be achieved in the absence of vision and how this compared to using vision alone or more traditional teaching methods. Furthermore, neither group considered the pedagogical issue of comparing the macro to the nano world. In this paper, we address the above issues by the design and implementation of a haptic feedback system interfaced with a nanoscale virtual environment that (1) provides for increased accessibility to a classroom environment, and (2) facilitates exploration and perception of nanoscale concepts to both sighted and visually impaired students.

\section{Method}

In order to get across educational concepts to students over a wide range of ages, our approach is to compare the nano world to something the student is familiar with, the everyday macro world. In order to encourage learning, the student is allowed to actively switch between the two different worlds in a virtual environment. The virtual environment consists of purely haptics, for students who are visually impaired, and a combination of haptics and a visual display for sighted students. The most important step in the methodology was to determine what are the "big ideas" that should be conveyed to the learner. This was performed through analysis of the literature and through personal knowledge. The next step was to select an appropriate haptic feedback mechanism that would be effective to use, as well as amenable to implementation in grade school or college classrooms. Then individual learning modules were developed to convey each of the core concepts.

\subsection{Educational Concepts to be Taught}

The two main concepts that we would like to communicate are: 1) an understanding of the size difference between the macro and nano worlds, and 2) an understanding of the dominant forces and their behavior as a function of scale. An important contribution to the latter is that, as the size of matter decreases to less than $100 \mathrm{~nm}$ in dimension, the surface to volume ratio significantly increases. As a result, at the nanoscale, surface properties dominate volume properties and surface forces can dominate inertial (macro) forces. Figure 2 shows a comparison of scales for the macro and nano worlds. 


\section{Scale of Macro-Nano}

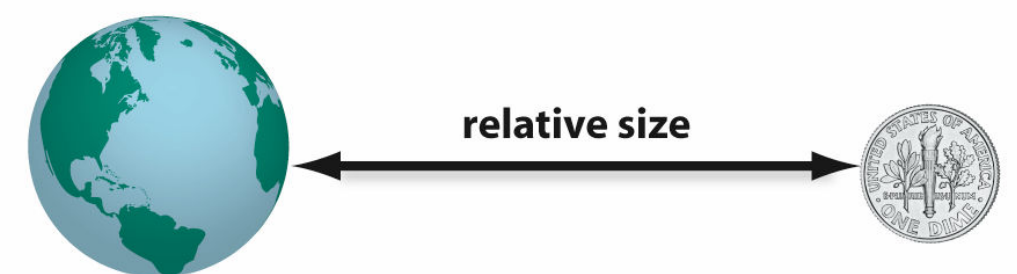

\section{Macro World Nano World}

Force $\quad \begin{aligned} & \text { human } \\ & \text { hair } \\ & (60-100 \mathrm{um})\end{aligned}$
Mass $\begin{aligned} & \text { dominate }(\sim \text { Newtons) } \\ & \text { bulk volume (atoms) dominate }\end{aligned}$
Physical properties largely based on continum
Chemical $\begin{aligned} & \text { atomic potentials, surface forces } \\ & \text { stable forces, low reactivity, low energy }\end{aligned}$

Figure 2. Comparison of scales and associated changes in properties.

The first of these concepts, the decrease in size, seems simple enough to communicate, however such phenomena have been found to be exceedingly non-intuitive and often poorly understood despite careful explanation ${ }^{3}$. Frequently students will view the macro world as one continuum and the sub-macro world on a different distinct scale, rather than one continuum. Light and his colleagues $^{3}$ suggest that visual experience, such as viewing sub-macro objects with scientific equipment, plays a key role in understanding the continuum of size from the macro to the nano level. It is likely that the key concept is one of controllably "zooming in" on different structures that is most effective and is likely to be successful in the haptic, in addition to the visual domain.

Both concepts will be portrayed through a virtual model of the scientific equipment of the nano world, an AFM, that changes in size between the different scales. AFM is a technique of imaging topographical features and measuring forces with atomic scale precision. It was developed in 1986 by Binnig, and his colleagues ${ }^{15}$, who worked at IBM's research lab in Zurich, Switzerland and later went on to win the Nobel Prize for his invention and achievements in atomic scale microscopy. The instrument consists of a cantilever probe and sharp tip (typically $>10 \mathrm{~nm}$ in 
size--see Figure 3) interacting with a surface. Use of a virtual AFM was chosen as AFM probes have contributed to much of our understanding of nanoscale surface structures and physical

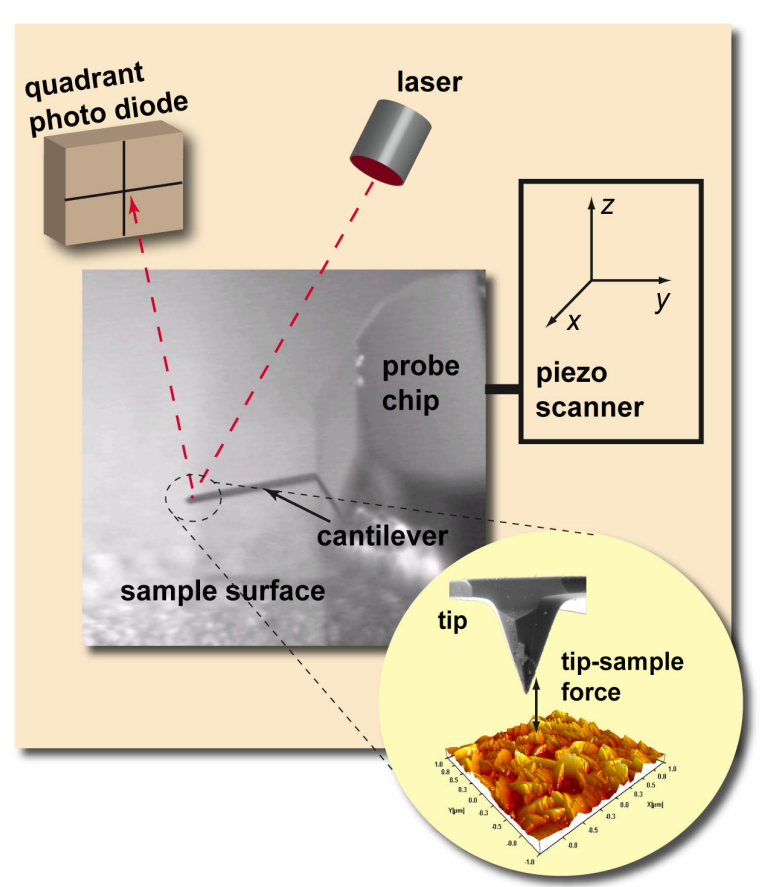

Figure 3. Diagram of an atomic force microscope system that uses the 'optical lever' approach to detect cantilever deflection. A laser is used to reflect light off of the back of the cantilever that is in contact with the surface. interactions.

For the second of the concepts to be taught: at the nanoscale, the cantilever probe of an AFM interacts with the surface of a material through a variety of tip-sample forces (the forces are discussed in section 2.2) that are modulated as a function of distance between the probe tip and surface. In the virtual reality simulation, the dimensions of the probe will be allowed to increase into the centimeter range, while holding the density of the tip material constant, in order to portray the changing of the dominant forces when moving to the macroscale,. While using this type of "zooming", students will be allowed to "turnoff" components of the net tip-sample force to enable understanding of the effects of individual types of forces.

\subsection{Physics of AFM Imaging and Force Spectroscopy}

The concept and physics upon which AFM is based is relatively simple. Essentially, a tiny probe in the form of a microscale cantilever beam (typically 100-200 um long, 20-30 um wide, and

1-3 um thick), with a sharp tip (radius $<10 \mathrm{~nm}$ ) located at its distal end, is used to interact with a sample surface of interest. As the cantilever is brought close to the surface $(<1 \mathrm{um})$, various interacting forces are encountered between the tip and sample surface, namely:

where,

$$
F_{\text {tip-sample }}=F_{\text {Lennard-Jones }}+F_{\text {capillary }}+F_{\text {electrical }}+F_{\text {magnetic }}+F_{\text {chemical }}
$$

$$
F_{\text {Lennard-Jones }}=F_{\text {repulsive }}+F_{\text {van der Waals }}
$$

These interacting forces are due to the interaction of atoms from the tip with those of the surface $^{16}$. The forces are of extremely small magnitude and are dominated by van der Waals and electrostatic (electrical) forces. van der Waals forces are weak secondary atomic bonding forces encountered between two atoms or molecules, such as between molecular chains of amorphous polymers. The energy of the van der Waal's potential is on the order of $1.6 \mathrm{eV}$ and the force is approximately $0.3 \mathrm{nN}$. The microscale dimensions of the cantilever beam (probe) of the AFM allow it to be sensitive enough to bend due to such small forces. In fact, cantilever probes have been designed that are sensitive down to picoNewtons of force. This extreme sensitivity to force is the primary reason that AFM is so extremely powerful-it can sense or detect atomic scale forces. 
Mathematically, the van der Waal's force between two atoms can be approximated as a function of the distance between the atoms $(z)$ as:

$$
F_{\text {van der Waals }} \approx \frac{1}{z^{6}}
$$

In the case of AFM, there are hundreds of atoms present at the probe tip and sample surface, thus the force for a flat sample area and spherical tip of radius $(R) \sim 10 \mathrm{~nm}$ is given by:

$$
F_{\text {van der Waals }}=\frac{A_{H} R}{6 z^{2}}
$$

where $z$ is the closest distance between the tip and sample surface and $A_{H}$ is the Hamaker constant (determined by the atomic polarizability, density, etc. of the sample material).

It should be noted that the force in equation (2) is attractive in nature. As the tip approaches the surface, at a certain critical separation distance, the tip will "flick" into contact with the surface. This "flick" instability occurs when the rate of increase of attraction, as the tip approaches the surface, exceeds the rate of increase of the restoring force of the deflected cantilever beam. The restoring force is dependent on the specific stiffness of the cantilever beam ${ }^{17}$. For cantilever probes of stiffness $k$ that can be approximated as a rectangular beam of width $w$, length $L$, Young's modulus $E$, and thickness $t$, the restoring force $F$ due to a deflection $x$ is given by:

$$
F=k x=\frac{E w t^{3}}{4 L^{3}} x
$$

At equilibrium the restoring force equals the attractive force:

$$
k x=\frac{A_{H} R}{6 z^{2}}
$$

If the deflection increases by $d x$ such that $z$ decreases an equal amount $(-d x=d z)$, instability occurs and the tip flicks into contact with the surface. Specifically, this condition occurs when:

$$
k d x=\frac{-A R}{3 z^{3}} d z=\frac{A R}{3 z^{3}} d x
$$

Thus, the critical distance for flick instability is given as:

$$
z=\left(\frac{A R}{3 k}\right)^{1 / 3}
$$

For typical contact mode cantilevers $(k=0.1-0.3 \mathrm{~N} / \mathrm{m})$ with a Hamaker constant on the order of $10^{-19} \mathrm{~J}$ (typical for ceramic and metal surfaces ${ }^{18}$ ), the critical distance is $\sim 2 \mathrm{~nm}$. Once contact of 
the tip is made with the surface, the tip is held in contact by adhesion forces. Upon retraction of the tip, the cantilever restoring (spring) force must overcome the adhesion forces before it comes out of contact. The adhesion force (a combination of van der Waal and electrostatic forces) encountered during retraction is considerably larger than the attractive van der Waal force encountered during approach-this behavior can be classified as approach-retract hysteresis ${ }^{18,19}$. It is important to note that the van der Waal force is the predominant surface force encountered by the probe tip near a surface thus it is fully elaborated upon here. The other components of the tip sample force in equation (1) are not elaborated here for the sake of brevity but full elaboration is given in the references ${ }^{16,18}$. Based upon the sensitivity of the cantilever deflection to tipsurface interacting forces, the measurement of forces between molecules or surfaces (force spectroscopy) as well as a complete mapping of surface topology (surface force microscopy) can be performed with atomic scale precision.

\subsubsection{Contact Mode AFM Imaging}

In contact mode AFM, the cantilever probe is rastered across the sample surface by piezo-driven or other active actuation schemes (or the sample stage is actuated). The force between the tip and sample is measured and held constant by monitoring the cantilever deflection with coupling to a control feedback system. The deflection is commonly measured by an optical lever detection scheme $^{20}$ (see Figure 3). In this scheme, the back of the cantilever tip is coated with a highly reflective metal thin film such as gold or aluminum; by reflecting a laser beam off the back of the cantilever and using a photosensitive detector (photodiode) to measure the change in position of the reflected light, the deflection of the cantilever is accurately measured. The deflection is a direct measurement of the force between the tip and sample. The signal of change in deflection from the photodiode is used as the input into the control system. This signal is used to regulate

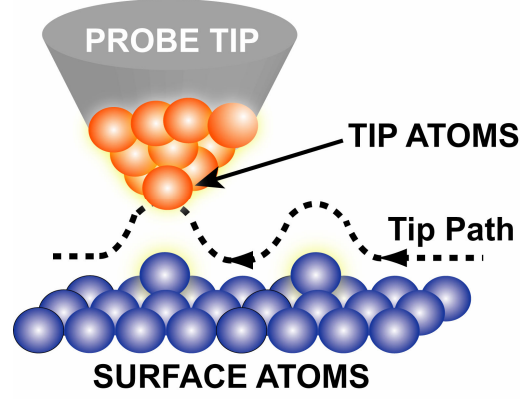

Figure 4. Description of the operation of an AFM. The probe tip follows the tip path to maintain constant height and force between the tip and sample. voltage applied to the piezoscanner that moves the cantilever to maintain a constant deflection (or distance between the tip and surface). Thus, the tip is made to follow the surface (see Figure 4). By plotting the error signal or amount of compensation by the piezo needed to maintain a constant cantilever deflection versus position on the sample surface, a topographical (height) mapping of the surface can be produced. The high sensitivity of the cantilever to small forces, the small size of the probe tip, and the sub-nanometer precision in actuation by the piezoscanner allow the AFM to produce high-resolution surface images - from upwards of 50 microns square down to atomic resolution. In our virtual reality simulation, an analog of contact mode AFM imaging is used to portray "zooming" of surface features and topology from centimeters to nanometers in dimension.

\subsubsection{AFM Force Spectroscopy}

The extreme sensitivity of the probe tip to sample forces allows it to measure forces as a function of the tip-sample separation with the output being a force spectrograph or force-distance curve as shown in Figure 5. 


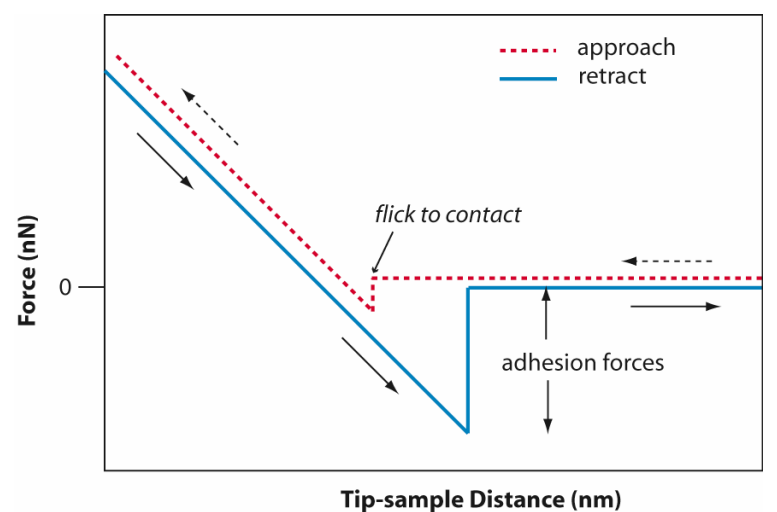

Figure 5. Diagram of a typical force-distance curve.

Force distance curves have been produced to measure adhesion forces ${ }^{21}$, protein conformation dynamics $^{22}$, and single molecule interactions ${ }^{18}$. We use an analog of the force spectroscopy mode to portray changes in force behavior as a function of scale.

\subsection{Choice of Haptic Device}

For use as instructional material for K-12 and undergraduate courses, the haptic feedback device chosen needs to be relatively inexpensive (preferably allowing for multiple devices per class), easy to set-up and use, and reliable. There are several different types of haptic feedback devices to choose from. Devices that provide vibratory feedback to the skin to convey information can be small, lightweight and very inexpensive, with either a single point of contact with the skin or multiple points. However, although this method may be appropriate for understanding the tapping mode of an AFM, its use is not very intuitive for representing surface topology or surface forces. Devices also exist that provide small scale (i.e., fingertip sized) shape information to the skin. These types of displays are more appropriate for describing geometric features but are limited in size and depth to a finger pad.

In order to get the feel of surface topologies that change greatly in depth or the surface to volume relationship of an object, a small scale shape display needs to be coupled with kinesthetic feedback. Kinesthetic feedback devices typically can provide force feedback in 3- (x, y and z) are 6-(x, y, z, roll, pitch, yaw) degrees of freedom (DOF). Together, a shape display and kinesthetic feedback would provide an effective means of portraying the haptics of the virtual world. Unfortunately devices that couple 3-DOF or 6-DOF force feedback devices with shape displays are only beginning to reach the prototype stage, and one cannot simply buy a shape display and mount it on a force feedback display (due to the relatively large size of the associated parts of a shape display compared to the moving tip of the force feedback display).

Although using only a force feedback display is less than ideal for providing geometric information, due to solely having a single point of contact with the virtual world, practically, it is the most appropriate solution. It should also be pointed out, although not necessarily desirable to replicate, that an AFM, when used, only has a single point of contact with a surface. Thus, in this sense, the haptic hardware closely simulates the real world. However, part of the resulting evaluation of the modules will test to verify that students were engaged rather than frustrated with the force feedback displays, particularly when only force feedback is available. 
In terms of the actual hardware, the current state-of-theart in the use of force feedback displays for nanotechnology has focused on the use of high-end $(>\$ 10,000)$ force feedback devices. More affordable force feedback joysticks have been considered (e.g., Jones and her colleagues ${ }^{12}$ ); however, they are velocity rather than position based. More recently, Novint Technologies has developed a 3-DOF position based force feedback device, the Novint Falcon (see Figure 6) which is affordable, as well as easy to set-up and use. We used this device for our implementation as it can be easily duplicated (multiple times) in the classroom.

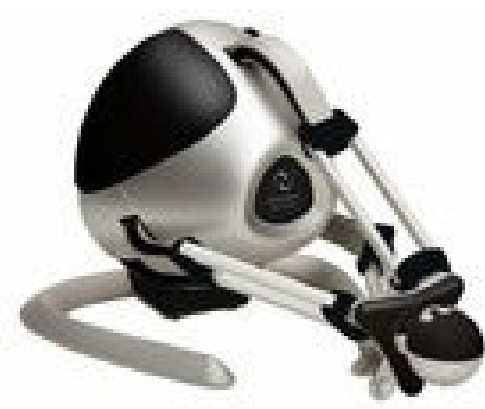

Figure 6. Photograph of the Novint Falcon.

Using the Novint Falcon, the learning modules were developed in $\mathrm{C}++$ on a $3.2 \mathrm{GHz} \mathrm{PC}$ using openGL for the visual rendering and Novint's software development kit for the haptics.

\subsection{Module 1: Concept of Size between Scales}

The objective of module 1 is to teach students that the macro and nano worlds are part of the same continuum, with more features potentially appearing as the scale of viewing decreases. In order to communicate this concept we have decided to allow students to controllably "zoom in" on objects they are familiar with, such as a compact disc or human hair. As the scale decreases, they will become aware of the details they can feel and, for the sighted students, see in the topology, while appreciating the fact that it is the same object.

For the actual implementation of the haptic feedback on the Novint Falcon, both the macro- and nanosurface topologies are simulated using an analog of an AFM - a relatively stiff virtual spring, is used to model the haptic contact of the probe with a virtual surface. The software works by loading gray-scale images of objects at different zooming factors for which the intensity of the image indicates the relative surface height of that point. For nanoscale images, AFM image files of 'real' material surfaces imaged in contact mode are used (see Figure 7).

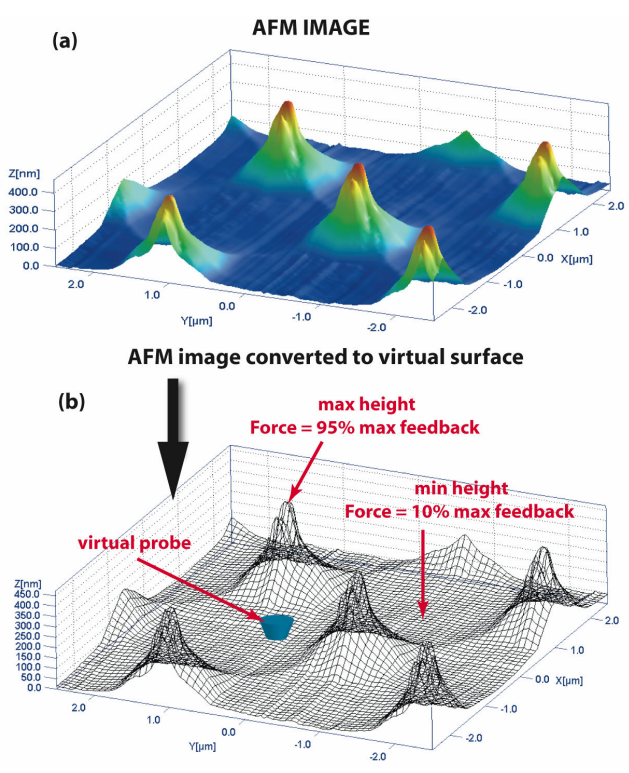

Figure 7. (a) 3-D AFM image is converted to (b) a gray scale image (shown here in wireframe for better representation). 
From each image, a 3-D perspective graphical representation of the topology is created in openGL, for use by sighted students, by performing triangular tessellation of the surface. The virtual cantilever probe appears as a sphere in the virtual environment, which the user controls via the Falcon force feedback device. As the user moves the virtual probe of the Novint Falcon over the virtual surface, the forces of interaction are rendered when contact is made. For an AFM, this force is equivalent to the force produced by the deflection of the cantilever, $\mathrm{F}=\mathrm{d}^{*} \mathrm{k}$, where $\mathrm{k}$ is the stiffness of the cantilever beam. For the simulation, these forces are a function of the penetration distance of the surface (see equation 9), which, alternatively, one could view as the amount of needed deflection compensation of the probe for it to remain on the surface. . Note that the forces are simulated with an appropriate scaling factor that incorporates the allowable signal bandwidth and sensitivity of the Falcon device.

$$
F=k_{\text {virtual }} * d_{\text {penetration }}
$$

In order to implement the force feedback algorithm, although more complicated algorithms are possible (e.g., Salisbury and his colleagues ${ }^{23}$ ), we used a simple volume based approach. For this algorithm, if the probe tip is located in the volume under a particular triangular surface patch, it is associated with the penetration of that particular surface. We first find which patch the center of the probe tip is located under and then calculate the shortest distance between the probe center and the surface of that patch. This can be done by using the following equation:

$$
d_{\text {penetration }}=\vec{v} \bullet\left(\vec{w}-\vec{p}_{i}\right)
$$

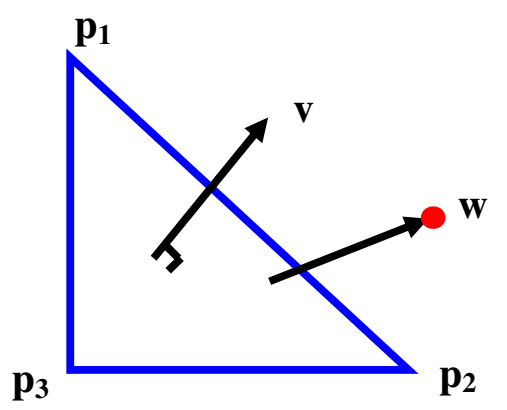

Figure 8. Example triangular surface patch.

where $\mathbf{v}$ is the normalized surface normal of the patch, $\mathbf{w}$ is the center of the probe tip and $\mathbf{p}_{\mathbf{1}}, \mathbf{p}_{\mathbf{2}}$ and $\mathbf{p}_{\mathbf{3}}$ are the corners of the triangular surface patch. If $\mathrm{d}_{\text {penetration }}$ is greater than zero, then the probe tip is not beneath the surface patch and the force should be set to zero. Otherwise, the probe tip is beneath the surface patch and the force magnitude will be as given in Equation 9 in the direction normal to the plane (given by the surface normal, v). The normalized surface normal is calculated by:

$$
\bar{v}=\frac{\left(\vec{p}_{2}-\vec{p}_{1}\right) \times\left(\vec{p}_{3}-\vec{p}_{1}\right)}{\left|\left(\vec{p}_{2}-\vec{p}_{1}\right) \times\left(\vec{p}_{3}-\vec{p}_{1}\right)\right|}
$$


In order to reduce the "bouncy" feeling when a large elevation change occurs, damping was added for the indentation phase of contact (but not the retraction phase). This improved the perception of the virtual topology when scanning over it.

\subsection{Module 2: Concept of Force Variation Across Scale Boundaries}

The objective of module 2 is to teach students about the changes in force behavior that occur as a function of scale-from macro to nano scales. This concept is taught through exploration of the changes in forces that dominate at each scale. At the macroscale, gravitational and inertial forces dominate, with gravity being simulated via a relatively constant feedback force applied to the virtual probe as a function of distance to the surface, and inertial effects simulated dependent on acceleration and the mass of the tip. At the nanoscale, surface forces dominate and are portrayed through the van der Waal interaction and approach-retract hysteresis. We modeled the changes in forces at the nanoscale via an analog of the AFM 'force spectroscopy' mode.

In this module, for the nanoscale world, a virtual force spectroscopy experiment is simulated with similar parameters and components as the module 1 simulation. However, the interaction forces are significantly modified. The interaction force modeled in the simulation is that of the van der Waal force as given in equation 4, where $z$ represents the distance between the probe tip and sample surface. In addition, the flick-to-contact instability is modeled according to equations 5-8 with the approach-retract hysteresis incorporated by making the adhesion (pull-off force) approximately 100 times greater than the flick-to-contact force.

The simulation is also capable of integrating other component forces of the net tip-sample force given in equation 5 such as the electrical (electrostatic) or chemical force components. This allows students the ability to 'turn on' or 'turn off' various components of the force to experience the contribution and behavior of each individual force.

\section{Preliminary Results and Discussion}

The virtual simulation and haptic interface has been demonstrated for several sighted students at various levels of education including elementary, undergraduate, and graduate students. Figure 9 shows an elementary school student using the simulation to traverse an AFM calibration sample. The overwhelming reaction to the simulation is extremely positive. Student comments include: "wow, this is cool", "how did they do that?", "this feels so real..." Students appear to be genuinely interested and excited by the ability to 'feel' a surface at various "zoom" levels, especially ones that they have some

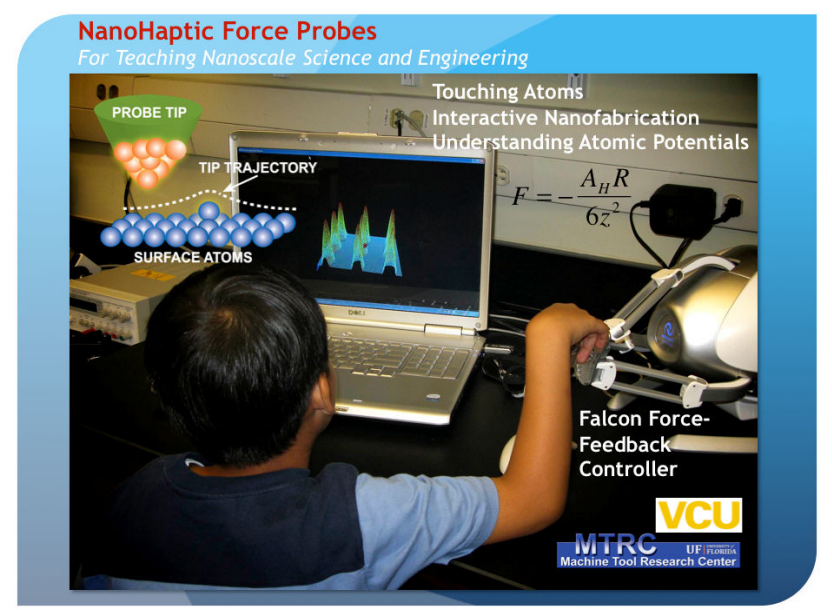

Figure 9. Photograph of elementary school student using the virtual haptic simulation. 
familiarity with such as a compact disc. The students are significantly engaged by the simulation. In addition, the students have felt that the simulation is intuitive and easy to operate.

Several faculty colleagues from various engineering departments have also been very impressed with the realism of the simulation and quickly point to the enormous possibilities to build different modules that capture fundamental physics, chemistry, and nanoscale concepts and phenomena. For example, we have plans to simulate nanoscale fabrication processes and tools for building nanostructures and nanoscale devices.

\section{Conclusion}

How things interact at the small scale of a nano-environment can be difficult for students to understand. This is particularly true for students who are visually impaired, as current pedagogical methods heavily rely on 2-D visual diagrams or molecular-scale images. In this paper we have introduced two teaching modules that will allow students to haptically interact with virtual worlds both at the nano-level and the macro-level, allowing all students a hands-on experience of the difference between these two worlds. The first module deals with explaining the concept of scale between the two worlds and how they are a continuum. The second module deals with explaining the concept of how different forces are dominant at the different scales, as they are dependent on the ratio of surface area to volume.

In the future, we intend to validate the use of these modules, in particular, for those students in grades 7-12, including students who are sighted and are blind or visually impaired. Grades 7-12 were chosen as students in these grades are old enough to verbalize their thoughts and the material is age appropriate to them. The two groups of students (sighted versus visually impaired) will be examined separately in order to discern whether a positive outcome occurs for both groups (rather than through an averaging effect). The two primary expected project outcome variables that we will examine are that the teaching modules will result in: (a) improved learning and retention of nanotechnology concepts, and (b) an increased interest in STEM fields (particularly nanotechnology) by these same students. As a control, we will compare our outcomes with the haptic learning modules with ones using other techniques, such as more traditional teaching methods. For these comparisons, time of exposure to the subject will be kept constant for the different methods.

The main assessment tool will be the use of pre-assessment and post-assessment questionnaires used with all learning groups. The pre-assessment will consist of two components, one to evaluate knowledge about nanotechnology before the teaching modules are used, and one to evaluate interest in nanotechnology and STEM fields in general. The post-assessment will contain the same questions as the pre-assessment with some additional questions. In terms of what they have learned, students will also receive questions to see how well they can transfer their knowledge to new but related situations (e.g., drug delivery). In terms of interest, students will also receive questions as to what they thought about the teaching modules, and whether they changed their opinions about nanotechnology and/or STEM fields. In addition, field notes will be taken during the instruction of the teaching modules to evaluate improvements in the learning process itself. In particular, as by Jones and her colleagues ${ }^{12}$ the number of questions that students ask (beyond how to use the Falcon), analogies used, affective words and haptic words used will be recorded. 
We expect our haptic modules will have a positive effect on learning nanoscience, particularly for students who are visually impaired and, otherwise, have limited access to this technology.

\section{References}

1. Sharma, G., Constantinos, M. and Ferreira, A. (2005). Virtual Reality and Haptics in Nano- and Bionanotechnology. In (eds.)M. Rieth and W. Schommers Handbook of Theoretical and Computational Nanotechnology, X, pp.1-33.

2. Stevens, S., Sutherland, L., Schank, P. and Krajcik, J. "The Big Ideas of Nanoscience". Accessed from http://www.nclt.us.news/news_docs/Big_Ideas_of_Nanoscience-draft2.pdf on Jan 12, 2009.

3. Light, G. et al. (2007). Understanding Undergraduate Students' Conceptions of a Core Nanoscience Concept: Size and Scale.

4. Okamura, A.M., Richard, C., Cutkosky, M.R. (2002). Feeling is Believing: Using a Force-Feedback Joystick to Teach Dynamic Systems. Journal of Engineering Education. 91 (3), 345-349.

5. Williams, R.L. II, Chen, M-Y. and Seaton, J.M. (2003). Haptics-Augmented Simple-Machine Educational Tools. Journal of Science Education and Technology, 12 (1), 1-12.

6. Gillespie, R.B., Hoffman, M.B., and Freudenberg, J. (2003). Haptic Interface for Hands-On Instruction in System Dynamics and Embedded Control. $11^{\text {th }}$ Symposium on Haptic Interfaces for Virtual Environment and Teleoperator Systems, 410-415.

7. Bowen, K. and O'Malley, M.K. (2006). Adaption of Haptic Interfaces for a LabVIEW-based System Dynamics Course. Symposium on Haptic Interfaces for Virtual Environment and Teleoperator Systems, March 25-26, Alexandria, Virginia.

8. Grow, D.I., Verner, L.N. and Okamura, A.M. (2007). Educational Haptics. AAAI 2007 Spring SymposiaRobots and Robot Venues: Resources for AI Education,

9. Williams, R.L. II, He, X., Franklin, T. and Wang, Shuyan. (2007). Haptics-Augmented Engineering Mechanics Educational Tools. World Transactions on Engineering and Technology Education, 6 (1), 1-4.

10. Sitti and Hashimoto (2003). Teleoperated Touch Feedback from the Surfaces at the Nanoscale: Modelling and Experiments. IEEE/ASME Transactions on Mechatronics, 8 (1), 1-

11. Jones, M.G., et al. (2003). Learning at the Nanoscale : The Impact of Students' Use of Remote Microscopy on Concepts of Viruses, Scale, and Microscopy. Journal of Research in Science Teaching, 40 (2), 303-322.

12. Jones, M.G., et al., (2005). Haptic Augmentation of Science Instruction : Does Touch Matter? In G.J. Kelley and R.E. Mayer (eds.) Learning. pp. 111-123.

13. Millet, G. et al. (2008). Improving Perception and Understanding of Nanoscale Phenomena Using Haptics and Visual Analogy. In M. Ferre (Ed.) Eurohaptics 2008, 847-856.

14. Marchi, F. et al., (2005). Interactive Learning of Nanophysics Phenomena. Recent Research Developments in Learning Technologies. 
15. Binnig, G., C.F. Quate, and C. Gerber, (1986). Atomic Force Microscope, Physical Review Letters.

16. Yacoot, A. and Ludger, K. (2008). Aspects of scanning force microscope probes and their effects on dimensional measurement. J. Phys. D: Appl. Phys. 41, pp. 1-46.

17. Tabor, D., and R.H.S. Winterton, (1968). Surface Forces: Direct Measurement of Normal and Retarded van der Waals Forces, Nature Vol. 219, pp. 2.

18. Butt, H.r., B. Cappella, and M. Kappa, (2005). Force measurements with the atomic force microscope: Technique, interpretation and applications , Surface Science Reports, Vol. 59, 2005, pp. 1-152.

19. Pethica, J.B., and W.C. Oliver, (1987). Tip surface interactions in STM and AFM, Physica Scripta.

20. Meyer, G., and N.M. Amer, (1988) Novel optical approach to atomic force microscopy, Applied Physics Letters Vol. 53, No. 12, pp. 1045-1047.

21. Burnham, N.A., O.P. Behrend, F. Oulevey, and G. Gremaud, (1997). How does a tip tap?, Nanotechnology

22. Vogel, V., (2006). " Mechanotransduction Involving Multimodular Proteins: Converting Force into Biochemical Signals", ANNUAL REVIEW OF BIOPHYSICS AND BIOMOLECULAR STRUCTURE.

23. Salisbury, K. et al., (1995). Haptic Rendering: Programming Touch Interaction with Virtual Objects. Symposium on Interactive 3D Graphics, Monterey CA USA, 123-130. 